\title{
Cellular model of neuronal atrophy induced by DYNC111 deficiency reveals protective roles of RAS-RAF-MEK signaling
}

\author{
Zhi-Dong Liu ${ }^{1}$, Su Zhang ${ }^{1,2}$, Jian-Jin Hao ${ }^{1}$, Tao-Rong Xie ${ }^{1}$, Jian-Sheng Kang ${ }^{1,2}{ }_{(\mathbb{D}}$ \\ ${ }^{1}$ Key Laboratory of Nutrition and Metabolism, Institute for Nutritional Sciences, Shanghai Institutes for Biological Sciences, \\ Chinese Academy of Sciences, Shanghai 200231, China \\ ${ }^{2}$ University of Chinese Academy of Sciences, Beijing 100049, China \\ $\triangle$ Correspondence: jskang@sibs.ac.cn (J.-S. Kang) \\ Received June 17, 2016 Accepted July 7, 2016
}

\begin{abstract}
Neuronal atrophy is a common pathological feature occurred in aging and neurodegenerative diseases. A variety of abnormalities including motor protein malfunction and mitochondrial dysfunction contribute to the loss of neuronal architecture; however, less is known about the intracellular signaling pathways that can protect against or delay this pathogenic process. Here, we show that the DYNC111 deficiency, a neuron-specific dynein intermediate chain, causes neuronal atrophy in primary hippocampal neurons. With this cellular model, we are able to find that activation of RAS-RAF-MEK signaling protects against neuronal atrophy induced by DYNC111 deficiency, which relies on MEK-dependent autophagy in neuron. Moreover, we further reveal that BRAF also protects against neuronal atrophy induced by mitochondrial impairment. These findings demonstrate protective roles of the RAS-RAF-MEK axis against neuronal atrophy, and imply a new therapeutic target for clinical intervention.
\end{abstract}

KEYWORDS RAS-RAF-MEK pathway, atrophy, dynein intermediate chain, mitochondria, hippocampal neuron, autophagy

Zhi-Dong Liu and Su Zhang have contributed equally to this work.

Electronic supplementary material The online version of this article (doi:10.1007/s13238-016-0301-6) contains supplementary material, which is available to authorized users.

\section{INTRODUCTION}

For normal aging, age-related brain atrophy is a mild process, responsible for increased risk of memory decline with increasing age (Pakkenberg et al., 2003; Fox and Schott, 2004), whereas the highly accelerated rate of brain atrophy has a major or sole role in neurodegenerative diseases (Regeur et al., 1994; Schott et al., 2003). Loss of neuronal architecture is the main contributor to brain atrophy (Swaab et al., 1994; Freeman et al., 2008), thus atrophy of brain and neuron can be well defined as the shortening or shrinkage of neurites. Neuritic atrophy is a common pathological feature of many neurodegenerative disorders including amyotrophic lateral sclerosis (ALS), Alzheimer's disease (AD), Parkinson's disease (PD), and Huntington's disease $(H D)$. A neuron consists of a soma and long neurites including one axon and multiple dendrites. The physiological structures of neurons render them particularly vulnerable to motor protein malfunction, protein aggregation, and mitochondrial dysfunction. Particularly, abnormalities to dynein and mitochondria are linked to ALS (Soo et al., 2011).

Cytoplasmic dynein is the main driving force for minusend-directed transport of cargos (Holzbaur and Vallee, 1994). Cytoplasmic dynein is a large protein complex $(\sim 1.5$ $\mathrm{MDa}$ ) containing heavy chains, intermediate chains, light intermediate chains, and light chains (Pfister et al., 2005). Mutations and 9-bp deletion of cytoplasmic dynein heavy chain (Dync1h1) are sufficient to cause neuron degeneration (Hafezparast et al., 2003; Chen et al., 2007; Banks and Fisher, 2008; Lipka et al., 2013). Mutations of cytoplasmic dynein light intermediate chain 2 (Dync1li2) and dynein intermediate chain (Dync1i) result in reduction of dendrite arborization of Drosophila neurons (Zheng et al., 2008; Boylan and Hays, 2002). However, less is known about the 
physiologic role of Dync1i in higher animals, especially the isoforms of Dync1i1, which are neuron-specific and not expressed in glia in the brain (Myers et al., 2007). Two genes (Dync1i1 and Dync1i2) in rodents encode cytoplasmic dynein intermediate chains, in which Dync1i1 is a neuronspecific gene (Fig. 1A). In line with previous reports (Myers et al., 2007), most mRNAs of the intermediate chain isoforms are expressed in rat brain, while the mRNA of Dync1i2C and Dync1i2B are expressed in most tissues (Fig. 1A). Here, we demonstrate that the knockdown of Dync1i1 causes neuronal atrophy and decreases mitochondrial motility in rat primary hippocampal neurons.

Using the cellular model of neuronal atrophy caused by DYNC1I1 deficiency, we are able to identify that RAS-RAFMEK signaling, but not PI3K-AKT signaling, protects neurons against dendritic atrophy in primary hippocampal neurons, and reveals that RAS-RAF signaling activates MEK-dependent protective autophagy. Moreover, we further demonstrate that BRAF can also protect dendrites from atrophy arisen from mitochondrial dysfunction. These findings of the RAS-RAF-MEK pathway for neuronal atrophy protection may provide a therapeutic target against the on-sets of neuronal atrophy.

\section{RESULTS}

\section{Knockdown of DYNC111 expression causes dendritic atrophy of primary hippocampal neurons}

Considering DYNC1I2 may play housekeeping function (Myers et al., 2007) and neurites of cultured neuron grow fast before 7 days in vitro (DIV7) (Dotti et al., 1988), we selectively knockdown DYNC1I1 expression at DIV6 in rat primary hippocampal neurons. Neurons transfected with shRNA1-6, a specific shRNA of Dync1i1 (Fig. S1A-F), show reduced dendritic complexity and shortened dendritic length (Fig. 1BD) compared to control neurons (Fig. 1E-G) at DIV11. For dendritic branches, the length of single branch seldom distributes beyond $350 \mu \mathrm{m}$ in control neurons or $200 \mu \mathrm{m}$ in DYNC1/1-knockdown neurons (Fig. 1H). Compared to the total dendritic lengths of control neurons $(1100 \pm 453 \mu \mathrm{m}, n=$ 75 , mean $\pm S D)$, the total dendritic lengths of DYNC1I1 knockdown neurons $(303 \pm 307 \mu \mathrm{m}, n=101$, mean \pm SD) are dramatically decreased $(P<0.001, t$ test) at DIV11 (Fig. 1I). Knockdown-resistant isoforms of Dync1i1 can partially rescue the phenotype caused by shRNA1-6 (Fig. S1C-H). The data suggest that the functions of DYNC1I1 in neuron are nonredundant and necessary for the maintenance of neuronal architecture.

The potential effects of shRNA1-6 on dendritic development or dendritic atrophy can both explain the reduced total dendritic lengths of DYNC1I1 knockdown neurons. To determine the effect of shRNA1-6, we have checked and quantified total dendritic lengths at different time points (1, 3, and 5 days) after shRNA1-6 transfection. As shown in
Figure 1. Knockdown of cytoplasmic dynein 1 intermediate chain 1 (Dync1i1) causes dendritic atrophy in primary hippocampal neurons. (A) The quantitativePCR results show the expression levels of cytoplasmic dynein 1 intermediate chains, including the isoforms of Dync1i1 (1A, 1B, 1C, and 1D) and the isoforms of Dync1i2 $(2 \mathrm{~A}, 2 \mathrm{~B}$, and $2 \mathrm{C})$ in $\mathrm{PO}$ rat hippocampus, cortex, heart, kidney, liver, and lung tissues. $(B-G)$ Representative neurons are transfected with shRNA1-6 (B-D, a specific shRNA of Dync1i1, see also Fig. S1) or Ctrl (E-G) at DIV6 and immunostained for the dendritic marker MAP2 (red in D and G) at DIV11. Green neurons are shRNA1-6 or Ctrl transfected neurons (green in $D$ and $G$ ). The scale bars represent $20 \mu \mathrm{m}$. (H) Sholl analysis for dendritic complexity of neurons transfected with control vector (Ctrl, $n=75)$ and Dync1i1 shRNA1-6 $(n=101)$. In control neurons, the maximum length of dendrite branches is found between 300 and $400 \mu \mathrm{m}$ from the cell body. The shRNA1-6 transfected neurons show marked shortened dendrites such that the majority of dendrites were located within 200 $\mu \mathrm{m}$ of the soma. Data are represented as mean \pm SEM. (I) Scatterplots with boxplots show that knockdown of DYNC1/1 expression causes dendritic atrophy. Primary hippocampal neurons are transfected with control vector (Ctrl, $n=26$, gray box), control shRNA (shRNA1-4, $n=26$, blue box) or shRNA1-6 ( $n=26$, red box) of Dync1i1 at DIV6. Following transfection, neurons are cultured additional 1,3 or 5 days before imaging and quantification of total dendritic length. The total dendritic lengths of control neurons slightly increase with increasing days in vitro. There is almost no length change of shRNA1-4 transfected neurons. However, the total dendritic lengths of shRNA1-6 transfected neurons are gradually and dramatically reduced at 3 and 5 days after transfection. ${ }^{*}, P<0.001$.

Fig. 1F, the distributions of total dendritic lengths show large variance. For 1,3 , and 5 days after transfection, the total dendritic lengths (in $\mu \mathrm{m}$, mean $\pm \mathrm{SD}$ ) of control neurons are $1075 \pm 330,1130 \pm 391$, and $1307 \pm 420$, respectively; for control shRNA1-4 transfected neurons, the lengths are 1037 $\pm 230,1010 \pm 234$, and $1006 \pm 310$; while the lengths of shRNA1-6 transfected neurons are $1041 \pm 340,535 \pm 119$, and $279 \pm 113$, respectively. These results are in line with reports that the dendritic architecture of neurons are mature at stage 4 (DIV4-7), while the maturation of synapses proceeds for following 2 weeks (stage 5) in vitro (Lalli, 2014). The data demonstrate that the DYNC1I1 knockdown by shRNA1-6 results in dendritic atrophy rather than dendriticdevelopment retardation (Fig. 1F). Thus, to address the question of whether there is an intrinsic signaling pathway for protecting neuron from atrophy, dendritic atrophy caused by DYNC1I1 knockdown in primary hippocampal neuron is a good cellular model of neuronal atrophy arisen by motor protein dynein malfunction. 
A

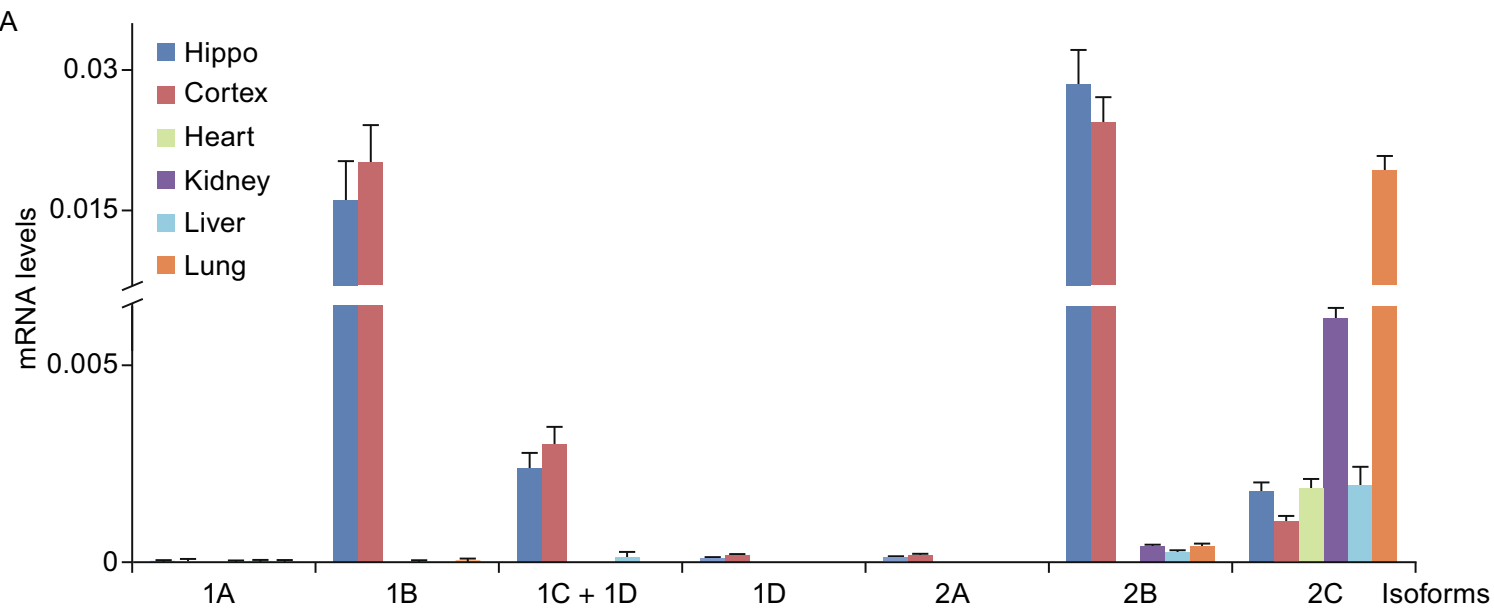

B

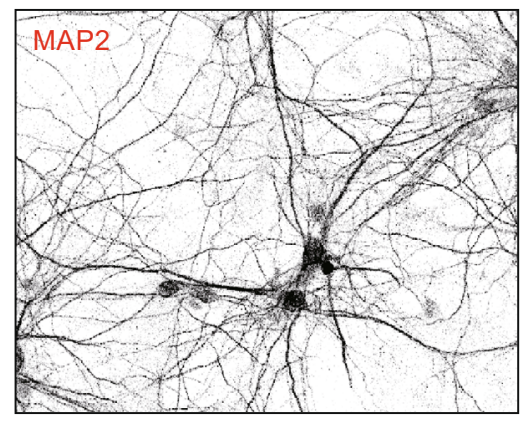

E
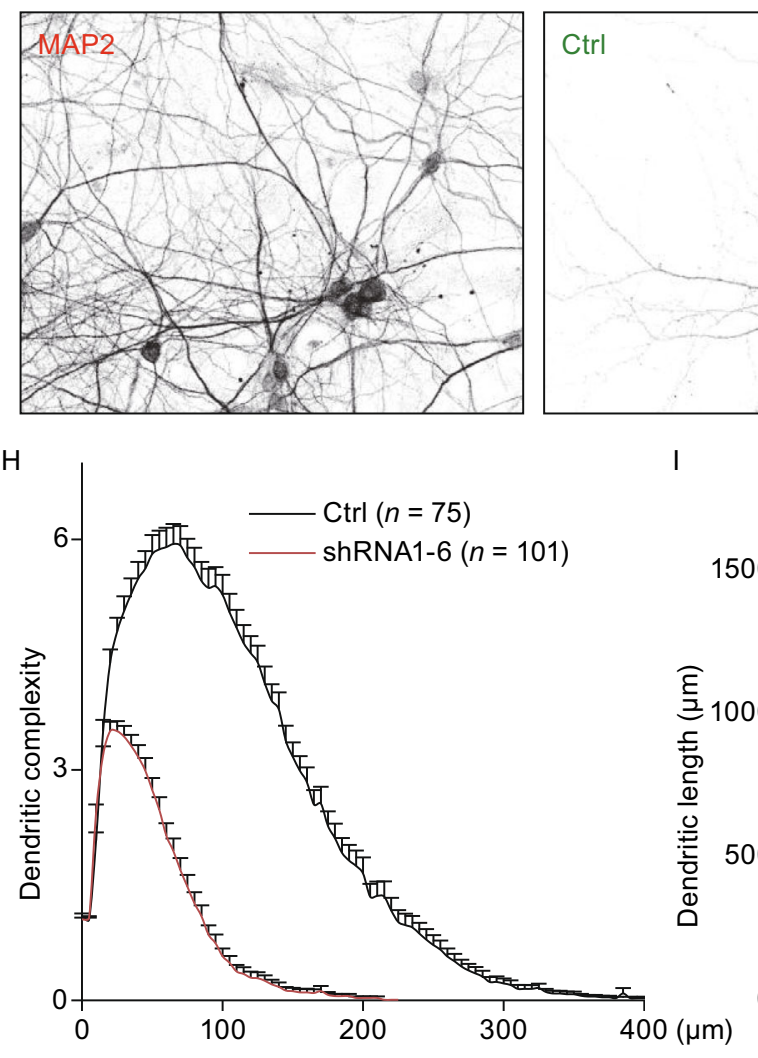

C

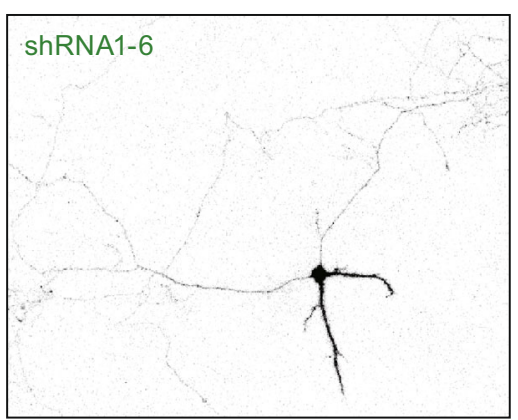

$\mathrm{F}$
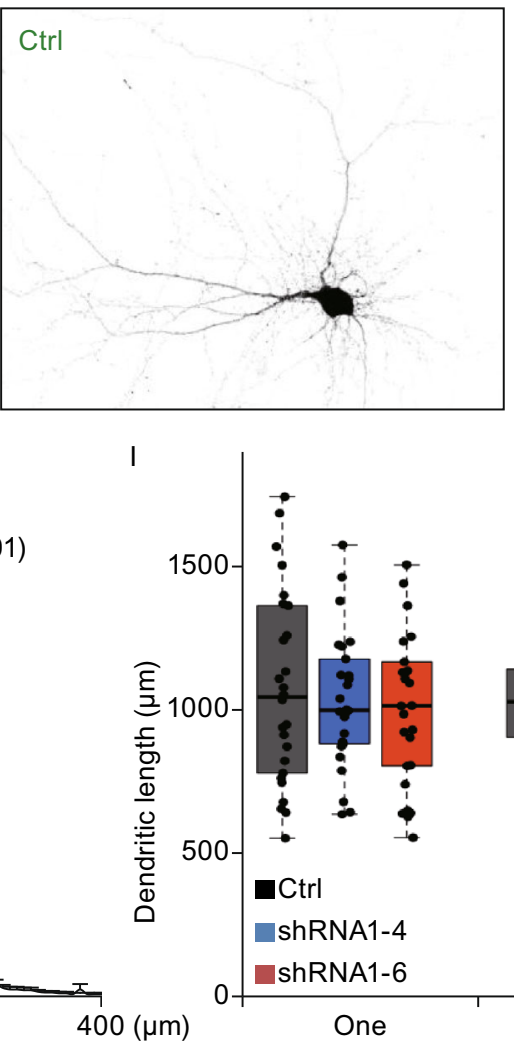

D

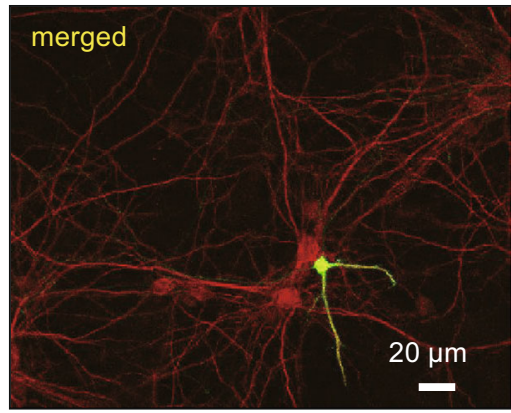

G
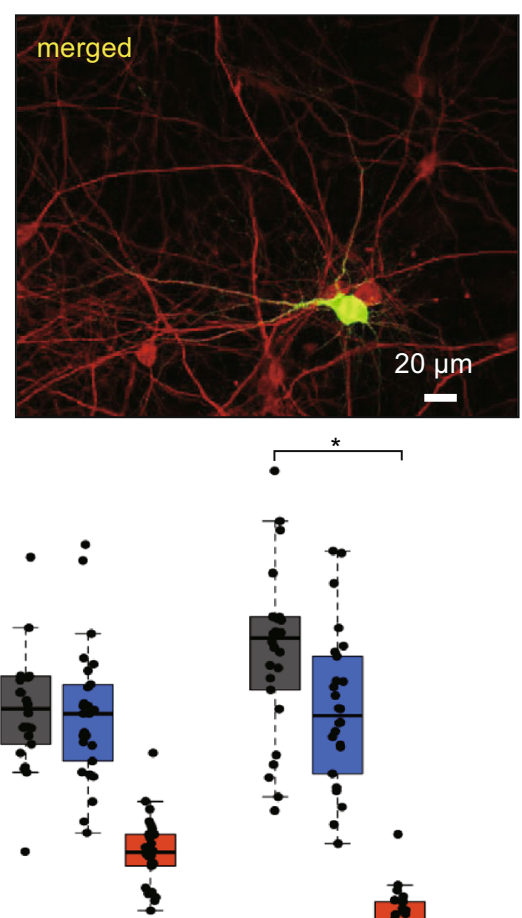

Three

day(s) 
BRAF protects against dendritic atrophy caused by DYNC111 knockdown in primary hippocampal neurons

For signaling pathways, we focus on the RAS-RAF-MEKERK mitogen activated protein kinase (MAPK) cascade and phosphoinositide 3-kinase (PI3K)-Protein kinase B (PKB, also known as AKT) pathways since they are both downstream signaling of neurotrophic factors (Chao, 2003). We overexpress AKT or BRAF in rat primary hippocampal neurons, where BRAF is the dominant functional RAF homolog in MAPK cascade and brain among three mammalian RAF proteins (ARAF, BRAF, and CRAF) (Galabova-Kovacs et al., 2006; Zhong et al., 2007). Consistent with the role in the signaling of neurotrophic factors, we have found that both BRAF and AKT overexpression can promote dendritic growth in control neurons (Fig. 2). The total dendritic lengths (in $\mu \mathrm{m}$, mean \pm SD) of control (Fig. $2 A$ ) and BRAF overexpressed neurons (Fig. 2B) are $1007 \pm 342$ and $1491 \pm 540$, respectively (Fig. 2F, gray and green boxes, $P<0.001$, $t$ test), and the lengths of control (Fig. 2G) and AKT overexpressed neurons (Fig. 2H) are $1021 \pm 323$ and $1581 \pm$ 567, respectively (Fig. 2L, gray and green boxes, $P<0.001$, $t$ test).

However, only BRAF can partially rescue dendritic complexity and protect against dendritic atrophy caused by DYNC1I1 knockdown (Fig. 2D-F), and the total dendritic lengths (in $\mu \mathrm{m}$, mean \pm SD) of DYNC1/1-knockdown neurons without (Fig. 2C) or with BRAF overexpression (Fig. 2D) are $264 \pm 135$ and $776 \pm 607$, respectively (Fig. 2F, red and purple boxes, $P<0.001, t$ test). Meanwhile, AKT overexpression has no effect on dendritic complexity and atrophy (Fig. 2J-L), and the total dendritic lengths (in $\mu \mathrm{m}$, mean \pm SD) of DYNC1I1-knockdown neurons without (Fig. 2I) or with AKT overexpression (Fig. 2J) are $330 \pm 197$ and $347 \pm 242$, respectively (Fig. $2 \mathrm{~L}, P=0.78, t$ test). Thus, the results suggest that RAF signaling may have important role in protecting against neuronal atrophy caused dynein malfunction.

\section{RAS-RAF-MEK signaling protects against dendritic} atrophy by activating MEK-dependent autophagy

To determine the signaling pathway for protecting dendritic atrophy, we use H-RAS mutants that activate RAF-MEK or/ and PI3K-AKT signaling selectively or non-selectively. Dominant active mutant RAS-G12V activates both RAF-MEK and PI3K-AKT signaling, while RAS-G12V-T35S mutant selectively activates RAF-MEK and the RAS-G12V-Y40C mutant selectively stimulates PI3K-AKT (Fiordalisi et al., 2001). In line with the above results of BRAF and AKT (Fig. 2), we have found that RAS-G12V-T35S mutant but not RAS-G12V-Y40C mutant has similar protective effect of BRAF on dendritic atrophy caused by DYNC1I1 knockdown (Fig. 3A-E). The total dendritic lengths (in $\mu$ m, mean $\pm S D$ ) of control (Fig. 3A), shRNA1-6 (Fig. 3B), RAS-G12V-T35S (Fig. 3C), and RASG12V-Y40C (Fig. 3D) transfected neurons are 1188 $\pm 228,377$
Figure 2. BRAF but not AKT overexpression protects against dendritic atrophy caused by DYNC1I1 deficiency in primary hippocampal neurons. (A-D) BRAF overexpression protects against dendritic atrophy caused by DYNC1I1 knockdown. Representative neurons are transfected with control vector (Ctrl) or shRNA1-6 cotransfected with or without BRAF at DIV6, and sequentially imaged at DIV11. The scale bars represent $20 \mu \mathrm{m}$. (E) Sholl analysis for dendritic complexity of neurons transfected with control vector combined without (Ctrl, $n=$ 37 , black) or with BRAF (Ctrl + BRAF, $n=48$, green) or Dync1i1 shRNA1-6 combined without ( $n=60$, red) or with BRAF (shRNA-6 + BRAF, $n=74$, purple). Data are represented as mean \pm SEM. (F) Scatterplots with boxplots show that BRAF overexpression promotes the dendritic growth of $\mathrm{Ctrl}$ neurons and protects dendritic atrophy caused by DYNC1I1 knockdown with shRNA1-6. (G-J) AKT overexpression has no effect on dendritic atrophy caused by DYNC1I1 knockdown. Representative neurons are transfected with control vector (Ctrl) or shRNA1-6 co-transfected with or without AKT at DIV6, and imaged at DIV11. The scale bars represent $20 \mu \mathrm{m}$. (K) Sholl analysis for dendritic complexity of neurons transfected with control vector combined without (Ctrl, $n=$ 26 , black) or with AKT (Ctrl $+\mathrm{AKT}, n=28$, green) or Dync1i1 shRNA1-6 combined without $(n=28$, red) or with AKT (shRNA-6 + AKT, $n=33$, purple). Data are represented as mean \pm SEM. (L) Scatterplots with boxplots show that AKT overexpression can also enhance the dendritic growth of Ctrl neurons, but fail to protect from dendritic atrophy caused by Dync1i1 knockdown with shRNA1-6. *, $P<0.001$.

$\pm 148,623 \pm 309$, and $351 \pm 234$, respectively (Fig. 3E). Compared to the length of shRNA1-6 group, the lengths of RAS-G12V-T35S group are improved ( $P<0.01, t$ test); RASG12V also plays protective role in dendritic atrophy by DYNC111 knockdown $(562 \pm 97 \mu \mathrm{m}$, mean $\pm \mathrm{SD}, P<0.01$, $t$ test), while RAS-G12V-Y40C has no effect ( $P=0.64, t$ test). These results suggest RAS-RAF pathway rather than PI3KAKT signaling can play protective role in neuronal atrophy caused by motor protein malfunction.

To confirm the role of RAS-RAF signaling in neuronal atrophy, we inhibit RAS-RAF-MEK signaling with pharmacological inhibitor trametinib, a specific inhibitor of MEK kinase (Yamaguchi et al., 2011). As shown in Fig. 3F-H, $100 \mathrm{nmol} / \mathrm{L}$ of trametinib blocks the protective effect of BRAF in dendritic atrophy induced by DYNC1I1 knockdown $(P<0.001, t$ test). For DYNC1I1-knockdown neurons at DIV11, the total dendritic lengths ( $\mu \mathrm{m}$, mean $\pm S D$ ) of BRAF overexpressed neurons without (Fig. 3F) or with $100 \mathrm{nmol} / \mathrm{L}$ of trametinib treatment (Fig. 3G) are $740 \pm 265$ and $234 \pm 112$, respectively (Fig. $3 \mathrm{H}$, purple and blue boxes), where the length of trametinib treated neurons with BRAF overexpression does not differ from the length of shRNA1-6 transfected neurons $(224 \pm 102 \mu \mathrm{m}$, mean 
A
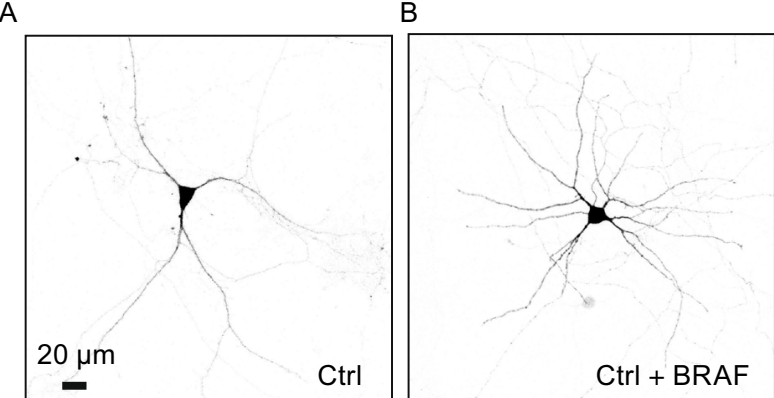

E

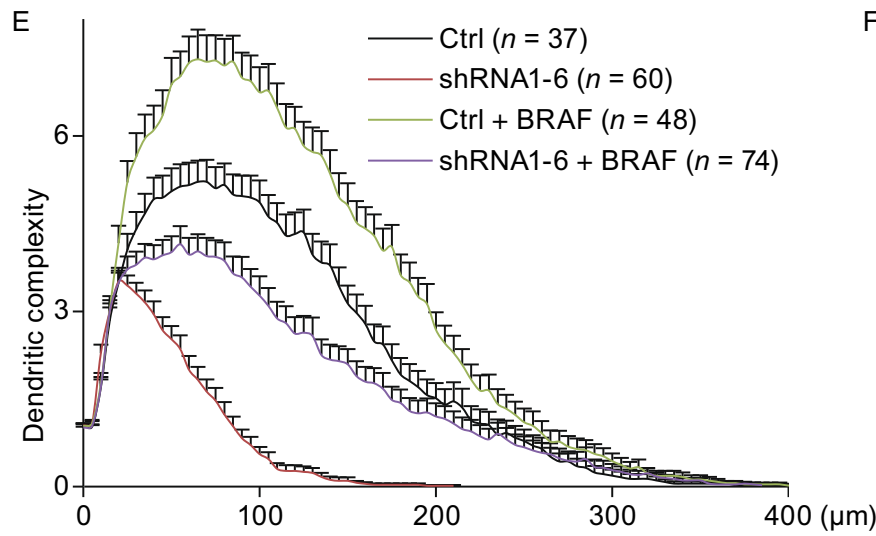

G
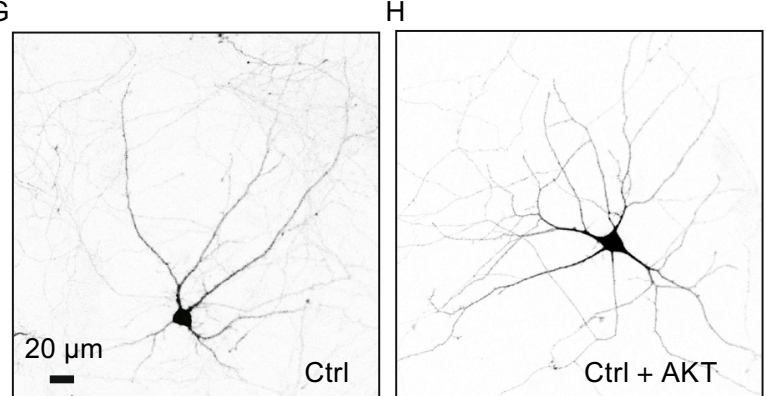

I

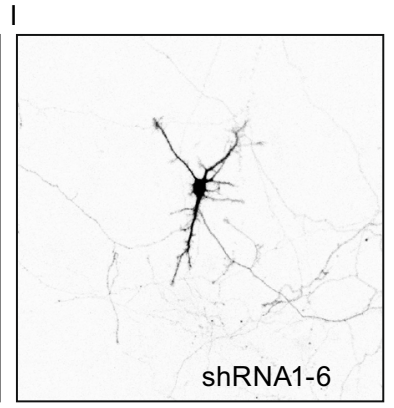

K

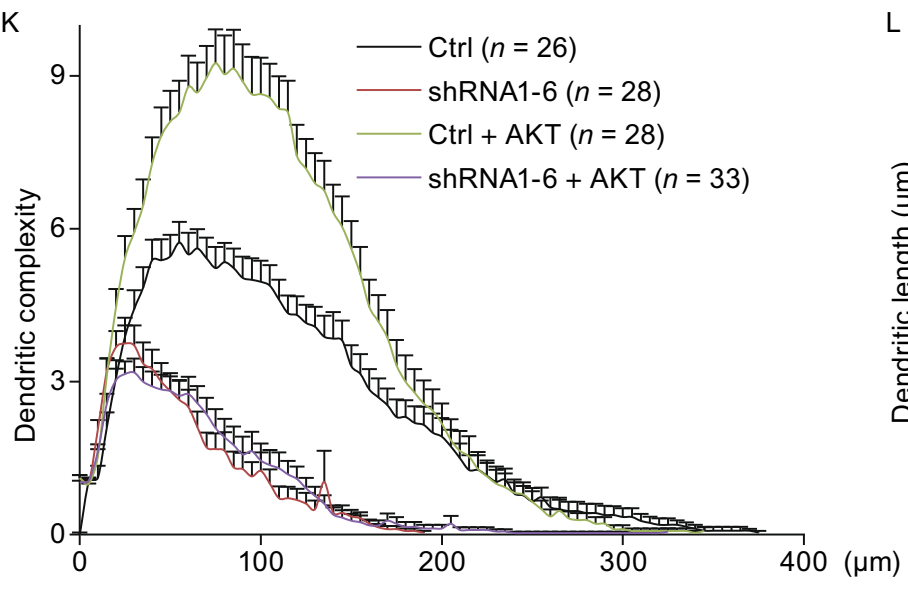

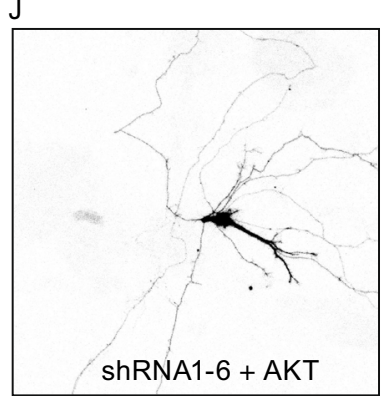
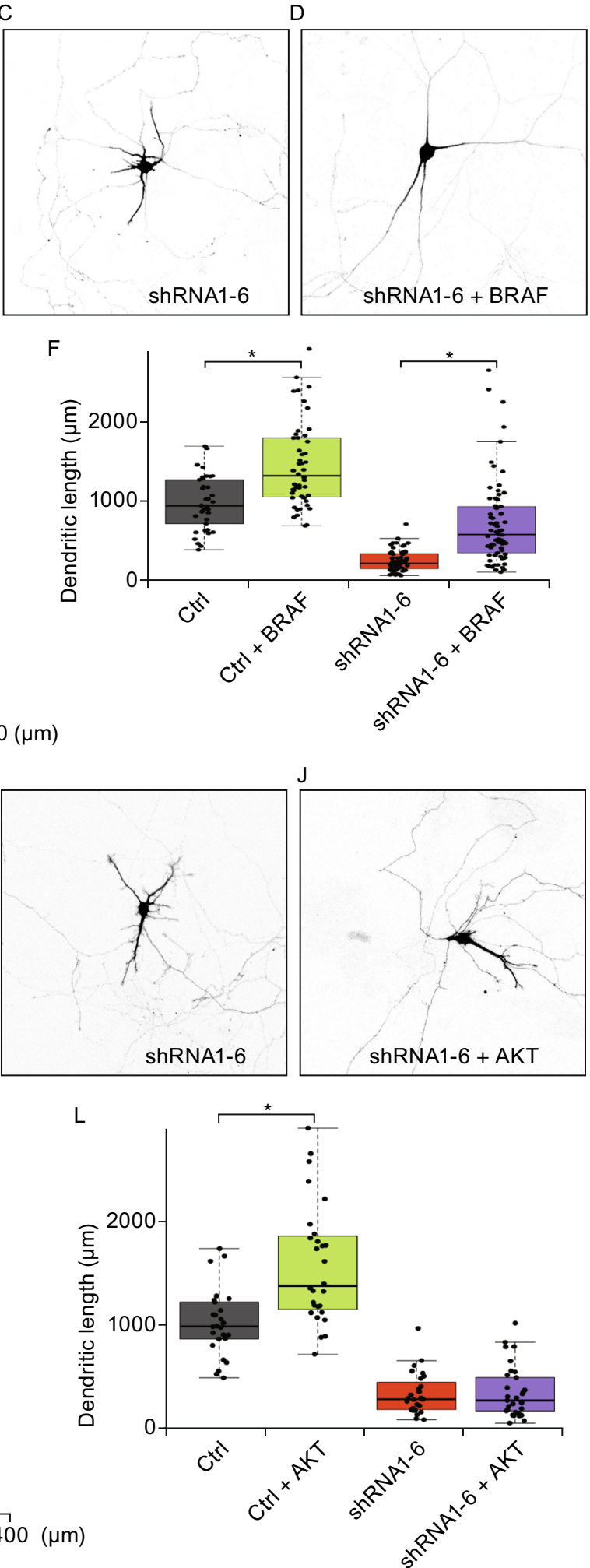
A

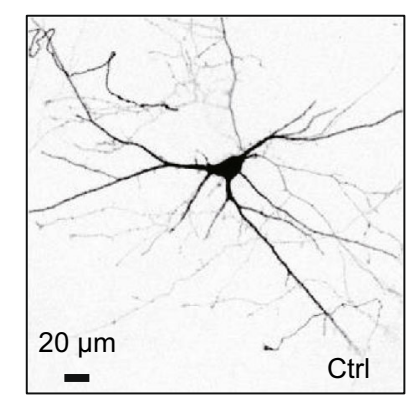

C

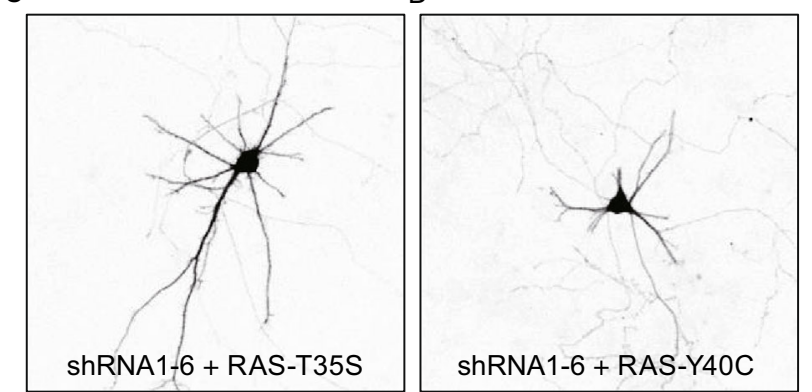

$\mathrm{F}$

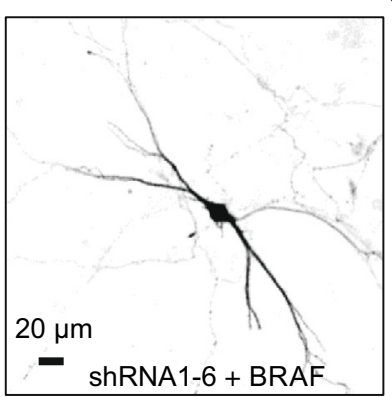

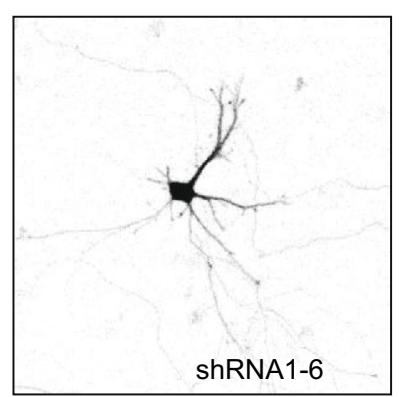

$\mathrm{D}$

G

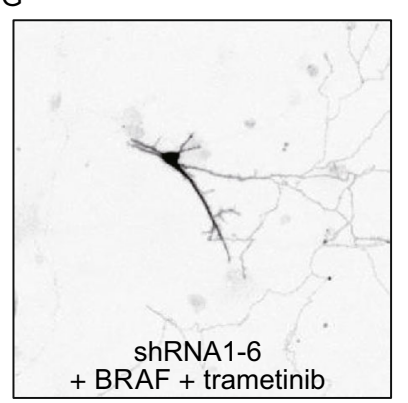

E

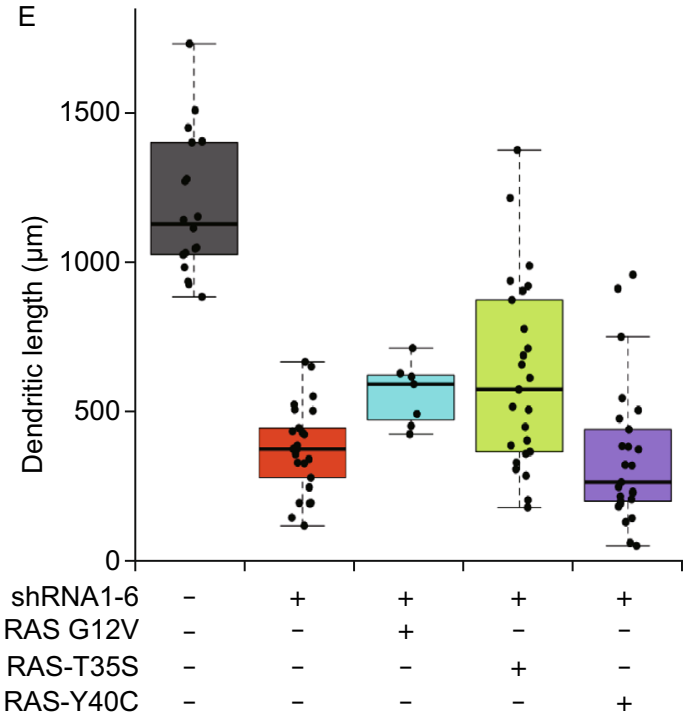

$\mathrm{H}$

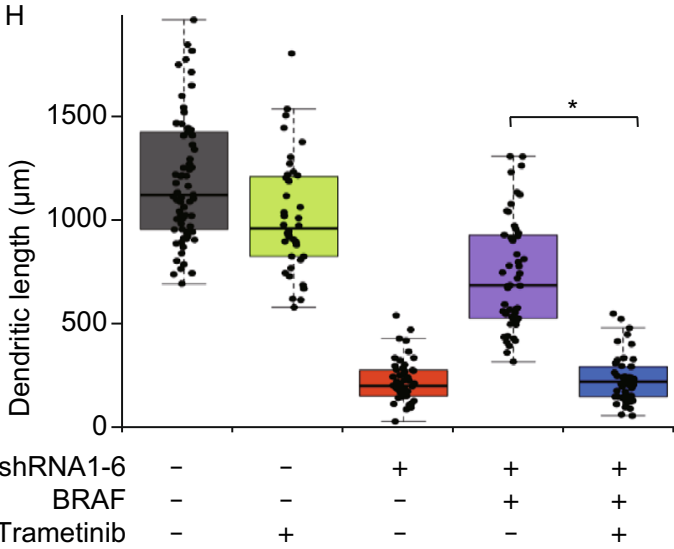

Figure 3. RAS-RAF-MEK signaling pathway protects against dendritic atrophy caused by DYNC1I1 deficiency. (A-D) Dominant active mutant G12V of RAS and selective RAF-activator mutant (G12V, T35S) of RAS protect against dendritic atrophy caused by DYNC111 knockdown, while selective AKT-activator mutant (G12V, Y40C) of RAS has no effect. Primary hippocampal neurons are transfected with control vector (Ctrl) or shRNA1-6 co-transfected with RAS-G12V, RAS-G12V-T35S or RAS-G12V-Y40C at DIV6, and imaged at DIV11. The scale bars represent $20 \mu \mathrm{m}$. (E) Scatterplots with boxplots show the total dendritic length distribution of neurons transfected with control vector (Ctrl, $n=18$, gray), shRNA1-6 ( $n=25$, red), shRNA1-6 with RAS-G12V ( $n=7$, light blue), RAS-G12V-T35S (RAS-T35S, $n=25$, green) or RAS-G12V-Y40C (RAS-Y40C, $n=25$, purple). (F and G) Trametinib, a specific inhibitor of MEK kinase in RAS-RAF-MEK signaling, inhibits the protective role of BRAF in dendritic atrophy caused by DYNC1I1 deficiency with shRNA1-6. Neurons are co-transfected with BRAF and shRNA1-6 at DIV6, and imaged at DIV11. The scale bars represent $20 \mu \mathrm{m}$. (H) Scatterplots with boxplots show the total dendritic length distribution of neurons transfected with control vector treated without ( $n=69$, gray) or with $100 \mathrm{nmol} / \mathrm{L}$ of trametinib ( $n=40$, green), shRNA1-6 ( $n=50$, red), shRNA1-6 cotransfected with BRAF treated without $\left(n=50\right.$, purple) or with $100 \mathrm{nmol} / \mathrm{L}$ of trametinib $\left(n=50\right.$, blue). ${ }^{*}, P<0.001$.

$\pm \mathrm{SD}, P=0.67, t$ test). Meanwhile, $100 \mathrm{nmol} / \mathrm{L}$ of trametinib slightly reduces the total dendritic lengths of control neurons (Fig. $3 \mathrm{H}$, gray and green boxes), where the lengths (in $\mu \mathrm{m}$, mean \pm SD) of neurons treated with or without trametinib are $1016 \pm 268$ and $1220 \pm 355$, respectively $(P<0.01, t$ test). Together, these results indicate that RAS-RAF-MEK signaling can play protective a role in dendritic atrophy caused by dynein malfunction.
BRAF protects against DYNC111 deficiency induced dendritic atrophy by activating MEK-dependent autophagy

Since dynein is required for autophagic clearance (Maday et al., 2012; Kimura et al., 2008; Ravikumar et al., 2005), dynein malfunction may cause protein aggregation and mitochondrial dysfunctions, and result in neuronal atrophy. 
Thus, we have checked whether RAS-RAF-MEK signaling can enhance autophagic function against atrophy in neuron. Indeed, the number of autophagosomes labeled with GFPLC3 is dramatically increased in DYNC1l1-knockdown neurons with BRAF overexpression (32 \pm 19 , Fig. $4 \mathrm{C}$ and $4 \mathrm{E})$, which is blocked by MEK inhibitor trametinib $(9 \pm 14, P<$ $0.001, t$ test, Fig. 4D and 4E). Whereas, the numbers of autophagosomes in control neurons treated without (Fig. 4A) or with trametinib, and DYNC1/1-knockdown neurons (Fig. 4B) are $7 \pm 6,5 \pm 5$, and $6 \pm 5$, respectively $(P=0.25$, one-way ANOVA test, Fig. 4E). These results suggest BRAF can activate MEK-dependent autophagy (Fig. 4C), which is necessary for the protective role of BRAF in DYNC1I1 deficiency caused neuronal atrophy (Fig. 3F-H). Moreover, the decreased number of autophagosomes in the presence of MEK inhibitor trametinib indicates that BRAF increases MEK-dependent autophagic influx and activity.

Additionally, to test whether the activity of increased autophagosomes is necessary for BRAF to play protective role in neuronal atrophy, we used lysosomal protease inhibitors (E64D and pepstatin A) to block autophagic clearance (Klionsky et al., 2012). As shown in Fig. 4F-J, blocking autophagic clearance inhibits the protective role of BRAF in neuronal atrophy. Again, BRAF shows robust role of protection in dendritic atrophy caused by DYNC1I1 knockdown. The total dendritic lengths (in $\mu \mathrm{m}$, mean $\pm \mathrm{SD}$ ) of DYNC1/1knockdown neurons with or without BRAF overexpression are $910 \pm 322$ and $233 \pm 101$, respectively (Fig. 4F-J). The protective role of BRAF in neuronal atrophy is blocked by 1 $\mu \mathrm{mol} / \mathrm{L}$ of E64D and pepstatin A treatment after transfection $(218 \pm 135 \mu \mathrm{m}$, mean $\pm \mathrm{SD}, P<0.001$ compared to BRAF protected neurons, $P=0.62$ compared to shRNA1- 6 transfected neurons, $t$ test, Fig. $4 \mathrm{I}$ and $4 \mathrm{~J}$ ). Thus, the results demonstrate that the activity of increased autophagosomes is necessary for the protective role of BRAF in neuronal atrophy. $1 \mu \mathrm{mol} / \mathrm{L}$ of inhibitors alone (E64D and pepstatin A) slightly reduces the total dendritic lengths of control neurons (Fig. 4J, green boxes), where the lengths (in $\mu \mathrm{m}$, mean \pm SD) of neurons treated with or without inhibitors are $1097 \pm 312$ and $1314 \pm 317$, respectively $(P=0.01, t$ test, Fig. $4 \mathrm{~J})$. The protective role of enhancing autophagic activity is also demonstrated with mTOR inhibitor rapamycin (Fig. S2), which also increase the number of autophagosomes in neurons (Fig. S3).

Lysosomal inhibitors can also increase the number of autophagosomes by blocking the function of lysosomal protease (Fig. S3F and S3G), but result in slightly reduced dendritic lengths (Fig. 4J). In addition, lysosomal inhibitors can slightly but not significantly increase the number of autophagosomes in BRAF protected shRNA1-6 transfected neurons (Fig. S3G), and block the protective role of BRAF in neuronal atrophy, which supports that lysosomal inhibitors block the activity of lysosomal enzymes to increase the number of autophagosomes in neurons. Whereas, BRAF increases the number of autophagosomes (Fig. 4C and 4E), enhances the activity of autophagy, and protects neuronal atrophy (Figs. 2D, 3F, and 4F), which are both MEK dependent and blocked by MEK inhibitor trametinib (Figs. 4D and $3 G$ ). Together, we reveal the protective function of RASRAF signaling in neuronal atrophy is mediated by activating MEK-dependent autophagy, which is protective and helpful against neuronal atrophy by cleaning protein aggregations and dysfunctional organelles, such as mitochondria.

BRAF also protects against dendritic atrophy caused by mitochondrial dysfunction in primary hippocampal neurons

Considering dynein is the major motor protein for cargo transport in dendrites (Kapitein et al., 2010), especially for mitochondrial transport, we have checked the motility of dendritic mitochondria (Fig. 5A-C). As shown in the kymograph of Fig. 5A (See also supplemental video 1), dendritic mitochondria show active motility in control neuron, while only a few dendritic mitochondria are motile in DYNC1I1knockdown neuron (Fig. 5B and supplemental video 2). To quantify the motility of dendritic mitochondria, motile and stationary dendritic mitochondria are separated with fast Fourier transform (FFT) algorithm (Fig. 5A and 5B). Considering motile mitochondria is prone to be ambiguous and overestimated due to the vague definition of motile mitochondria and the effect of photobleaching, we have quantified and compared the absolutely stationary mitochondria (The third row in Fig. 5A and $5 \mathrm{~B}$ ). The results demonstrate that stationary pool of dendritic mitochondria $(75 \% \pm 6 \%$, mean \pm SD in percentage) in DYNC1I1-knockdown neurons is significantly increased (versus $68 \% \pm 9 \%$ in control neurons, $P<0.001$, $t$ test, Fig. 5A-C).

Illustrated in Fig. 5A-C, DYNC111 knockdown decreases mitochondrial motility, which may result in accumulation of dysfunctional mitochondria (Maday et al., 2012; Xie et al., 2015). In turn, the accumulation of dysfunctional mitochondria might be an important or major factor to cause neuronal atrophy. To inspect this, we used a specific inhibitor (tetramethylrhodamine ethyl ester, TMRE) of mitochondrial function (Scaduto and Grotyohann, 1999), which is a frequently used mitochondrial marker. As shown in Fig. 5D-J, compared to the total dendritic lengths (in $\mu \mathrm{m}$, mean $\pm \mathrm{SD}$ ) of control neurons treated with relevant DMSO solution (944 \pm 278 , Fig. $5 \mathrm{D}$ and $5 \mathrm{H}$ ) or a non-inhibitory mitochondrial marker (tetramethylrhodamine methyl ester, TMRM) at $50 \mathrm{nmol} / \mathrm{L}$ (Scaduto and Grotyohann 1999) $(818 \pm 234$, Fig. $5 \mathrm{H}$, blue box), neurons treated with $50 \mathrm{nmol} / \mathrm{L}$ of TMRE show shortened dendritic lengths $(421 \pm 229, P<0.001, t$ test, Fig. $5 \mathrm{E}$ and $5 \mathrm{H}$, red box). Thus, mitochondrial dysfunction is sufficient to result in neuronal atrophy.

Moreover, since both dynein and mitochondria are therapeutic targets in neurodegeneration (Eschbach and Dupuis, 2011; Banks and Fisher, 2008; Moreira et al., 2010), we are also wondering whether BRAF can protect against dendritic atrophy induced by mitochondrial dysfunction. For the total dendritic lengths (in $\mu \mathrm{m}$, mean $\pm \mathrm{SD}$ ) of BRAF 
A
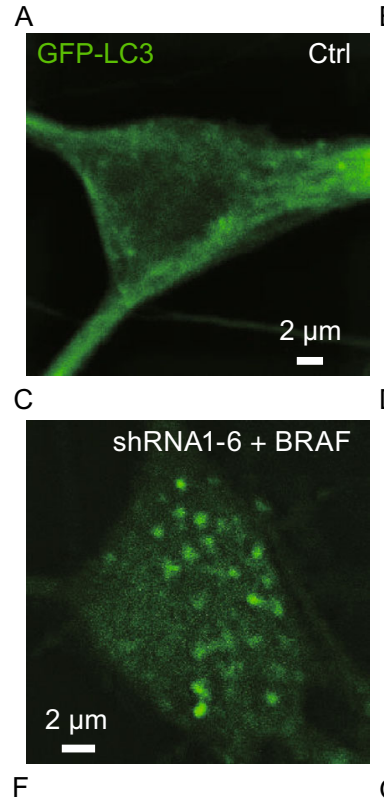

$\mathrm{F}$

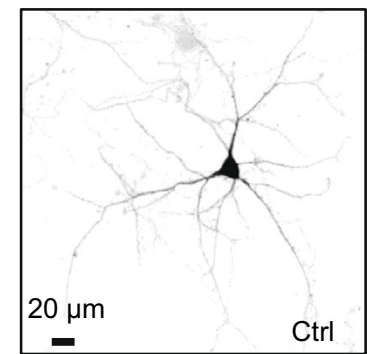

$\mathrm{H}$

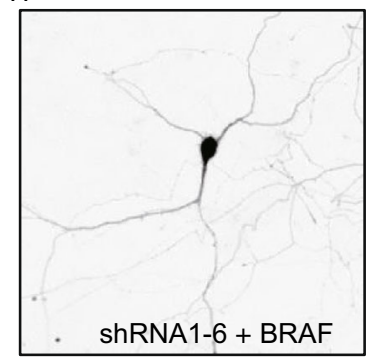

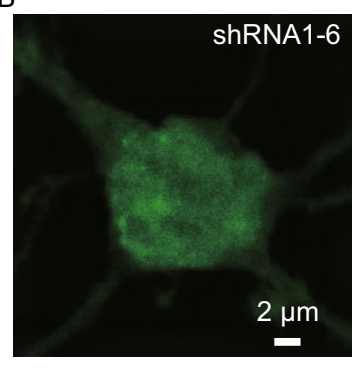

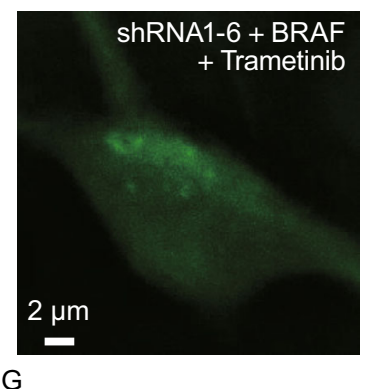

G

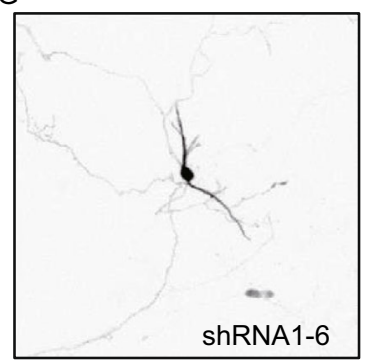

I

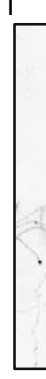

ShRNA1-6

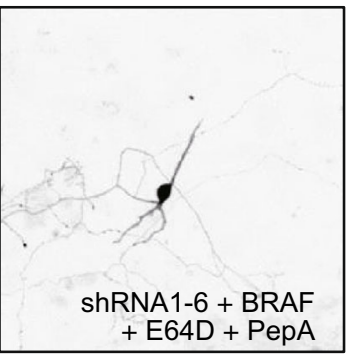

E

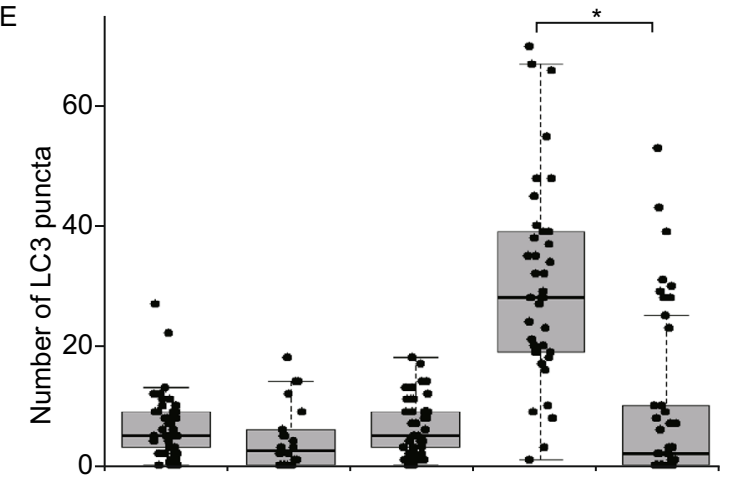

shRNA1-6

BRAF

Trametinib

J

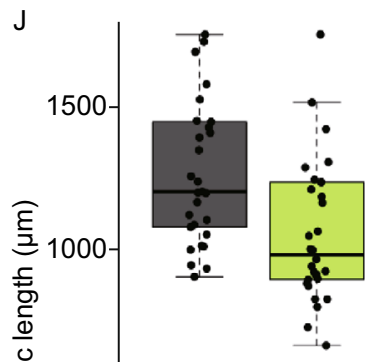

Figure 4. BRAF overexpression protects against DYNC1l1 deficiency induced dendritic atrophy by activating MEKdependent autophagy. (A-D) BRAF overexpression enhances protective autophagy, which is MEK dependent. Primary hippocampal neurons are co-transfected GFP-LC3 with blank vector, shRNA1-6, shRNA1-6 and BRAF treated without or with 100 $\mathrm{nmol} / \mathrm{L}$ of MEK inhibitor trametinib at DIV6, cultured additional 5 days and imaged at DIV11. Autophagosomes (green puncta) are labeled with GFP-LC3. All photos of GFP-LC3 are imaged with confocal microscope and further deconvolved for clarity. The scale bars represent $2 \mu \mathrm{m}$. (E) Scatterplots with boxplots show the number distribution of GFP-LC3 puncta in the soma of neurons transfected with control vector treated without $(n=41)$ or with $10 \mathrm{nmol} / \mathrm{L}$ of trametinib $(n=22)$, shRNA1-6 ( $n=48)$, shRNA1-6 cotransfected with BRAF treated without $(n=39)$ or with $100 \mathrm{nmol} / \mathrm{L}$ of trametinib $(n=44)$. (F-I) Lysosomal protease inhibitors (E64D and pepstatin A) can inhibit the protective role of BRAF in dendritic atrophy caused by DYNC1/1 knockdown with shRNA1-6. Neurons are transfected and treated without or with $1 \mu \mathrm{mol} / \mathrm{L}$ of E64D and pepstatin A at DIV6, cultured additional 5 days and imaged at DIV11. The scale bars represent $20 \mu \mathrm{m}$. (J) Scatterplots with boxplots show the total dendritic length distribution of neurons transfected with control vector treated without $(n=29$, gray) or with $1 \mu \mathrm{mol} / \mathrm{L}$ of E64D and pepstatin A $(n=30$, green), shRNA1-6 ( $n=30$, red), shRNA1-6 co-transfected with BRAF treated without $(n=30$, purple) or with $1 \mu \mathrm{mol} / \mathrm{L}$ of E64D and pepstatin A $(n=30$, blue). *, $P<$ 0.001 . 
A

A

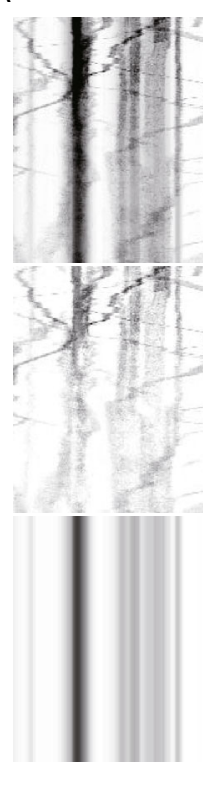

D
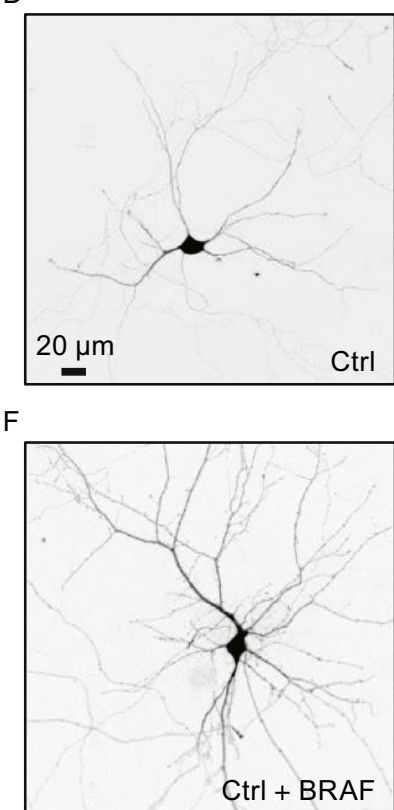

Ctrl

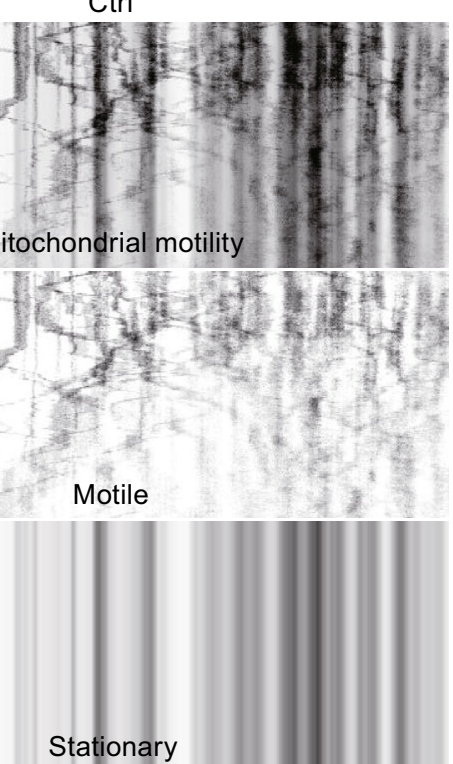

E

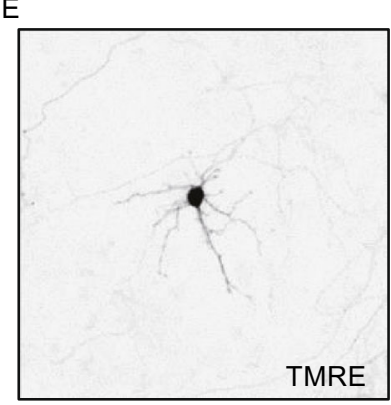

G

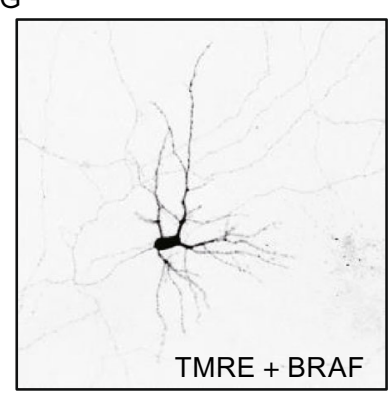

B shRNA1-6
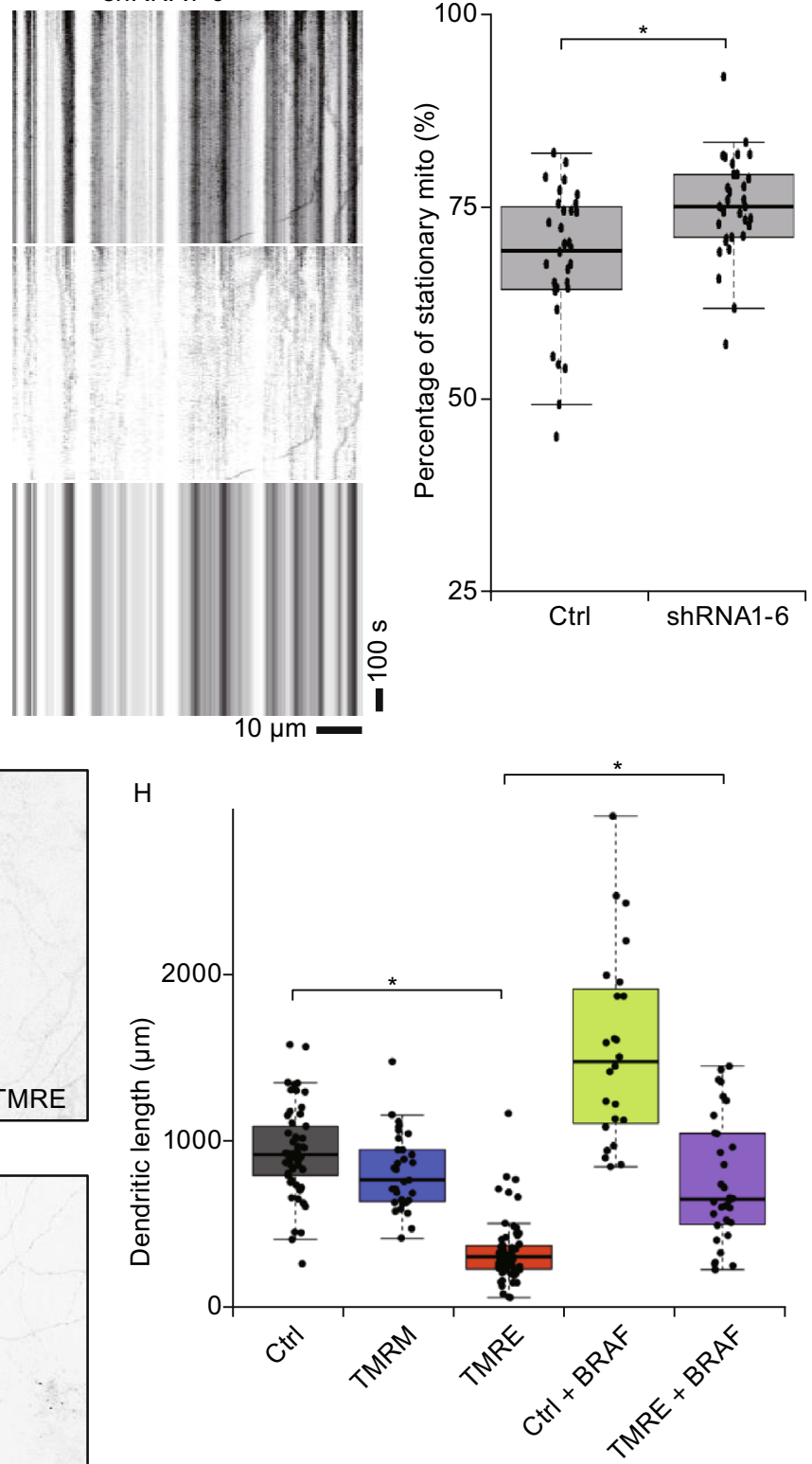

C

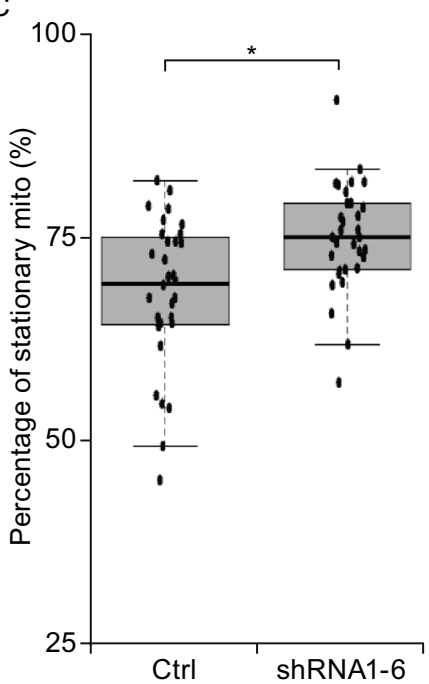

Figure 5. BRAF overexpression also protects against dendritic atrophy caused by mitochondrial dysfunction in primary hippocampal neurons. (A and B) Dync1i1 Knockdown decreases dendritic mitochondrial motility in primary hippocampal neurons. For clarity and quantification, motile and stationary dendritic mitochondria are separated with fast Fourier transform (FFT)algorithm. Kymographs show active mitochondrial motility in control neuron (A, Ctrl) and a few motile dendritic mitochondria in DYNC1/1-knockdown neuron (B, shRNA1-6). In kymographs, vertical lines represent stationary mitochondria, and slant lines or curves indicate motile mitochondria. Neurons are co-transfected at DIV6 with DsRed-mito and control vector (A) or shRNA1-6 (B), imaged at DIV11 and sequentially analyzed. The scale bars represent $10 \mu \mathrm{m}$ (x axis) and 100 seconds (y axis). (C) Scatterplots with boxplots show the percentage distribution of stationary dendritic mitochondria in neurons transfected with control vector $(n=32)$ or shRNA1-6 $(n=32)$. (D-G) BRAF overexpression also protects against dendritic atrophy caused by mitochondrial dysfunction. Neurons are transfected with control vector (Ctrl) or BRAF treated with control solution or with $50 \mathrm{nmol} / \mathrm{L}$ of specific mitochondrial function inhibitor TMRE at DIV6, cultured additional 5 days and imaged at DIV11. The scale bars represent $20 \mu \mathrm{m}$. $(\mathrm{H})$ Scatterplots with boxplots show the total dendritic length distribution of neurons transfected with control vector treated with vehicle (Ctrl, $n=53$, gray), with $50 \mathrm{nmol} / \mathrm{L}$ of TMRM $(n=31$, blue) or $50 \mathrm{nmol} / \mathrm{L}$ of TMRE $(n=63$, red), or transfected with BRAF treated without ( $n=24$, green) or with $50 \mathrm{nmol} / \mathrm{L}$ of TMRE ( $n=32$, purple). ${ }^{*}, P<0.001$. 


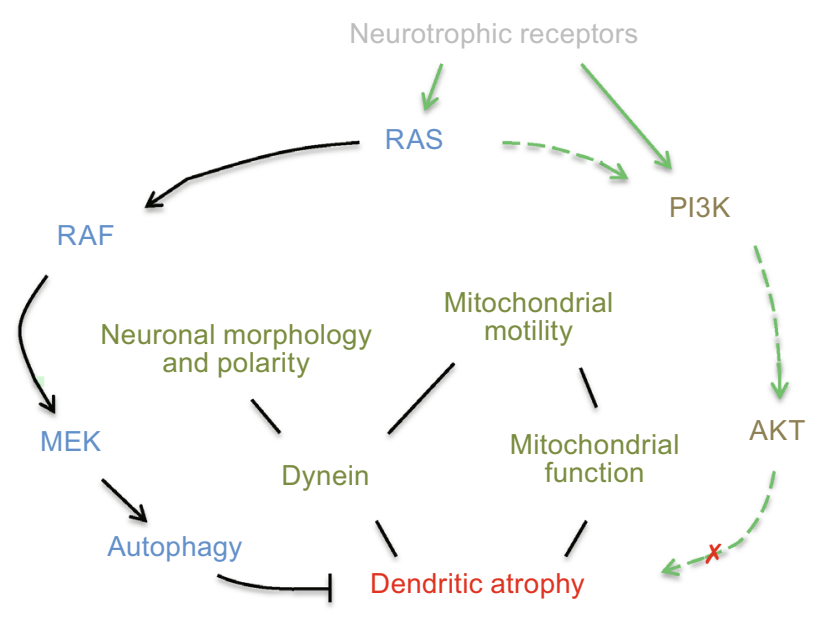

Figure 6. Schematic summary of general roles of RAS-RAFMEK pathway in protecting dendritic atrophy caused by dynein malfunction or mitochondrial dysfunction.

overexpressed neurons, BRAF promotes the dendritic growth of non-treated neurons $(1570 \pm 229$, Fig. $5 \mathrm{~F}$ and $5 \mathrm{H})$, similar as shown in Fig. 2B and 2F; BRAF overexpressed neurons are somewhat resistant to TMRE treatment, and protect dendrites from atrophy induced by functional inhibition of mitochondria ( $775 \pm 607$, Fig. $5 \mathrm{G}$ and $5 \mathrm{H}$, purple box). The total dendritic lengths of BRAF overexpressed neurons are significantly improved $(P<0.001, t$ test) compared to the lengths of TMRE treated control neurons (Fig. $5 \mathrm{H}$ red box). Together, these results based on above cellular models indicate that BRAF has a general protective role in neuronal atrophy caused by dynein malfunction or mitochondrial impairment.

\section{DISCUSSION}

Dynein is not only the major motor protein for dendritic cargo transport (Kapitein et al., 2010) and dendritic mitochondrial motility (Fig. 5), but also plays important roles in axonal structures and polarities (Song et al., 2009; Zheng et al., 2008). DYNC1/1 knockdown causes abnormal ER distribution in axon (Fig. S4), which is in line with the reported gatekeeper function of dynein (Song et al., 2009; Zheng et al., 2008). As an important cargo binding subunit of dynein (Ha et al., 2008), dynein intermediate chains (DYNC1I1 and DYNC1/2) are necessary for the functional integrity of dynein (Figs. 5 and S4) and the maintenance of neuronal architecture (Fig. 1). In addition, functions of both DYNC1/1 and DYNC112 are regulated by phosphorylation. The phosphorylation of DYNC1/1 serine 83 or DYNC1/2 serine 84 inhibits dynein intermediate chain binding to dynactin or paxillin (Vaughan et al., 2001; Rosse et al., 2012); while the phosphorylation of DYNC1/1 serine 80 or DYNC1/2 serine 81 is MAP kinase ERK1/2 dependent and can strengthen dynein activity in signaling cargos transport (Mitchell et al., 2012). In our study, we cannot exclude that the protective role of RASRAF-MEK signaling in neuronal atrophy may be partially due to upregulating the phosphorylation of DYNC1/2 serine 81 and thus partially compensate the dynein malfunction by DYNC111 knockdown, although we fail to detect any change of phosphorylation of DYNC112 serine 81 for control and DYNC111 knockdown (data not shown) in primary cultured hippocampal neuron, which may be explained by the low transfection efficiency of primary neuron and the expression of DYNC112C in glia.

\section{Protective role of RAS-RAF-MEK axis in neuronal atrophy caused by dynein malfunction}

In corroboration with our results, transgenic activation of RAS in neurons promotes neuronal growth and protects from lesion-induced degeneration (Heumann et al., 2000), but the mechanism is unknown; in addition, selective activation of BRAF can provide neuroprotection both in vitro and in vivo although it is not MEK dependent (Chin et al., 2004). Here, we identify that RAS-RAF signaling protects neurons against dendritic atrophy arisen from dynein malfunction, which relies on MEK-dependent autophagy (Fig. 4). MEK-dependent autophagy can be either protective or destructive autophagy (Wang et al., 2009) in non-neuronal cells. The role of MEK-dependent autophagy in neuron is unknown yet. Trametinib treatment and BRAF in control neurons doesn't affect the numbers of autophagosomes (Figs. 4 and Fig. S3E), which suggests that MEK-dependent autophagy has minor role in neuron under normal condition. However, we demonstrate that RAS-RAF pathway activates protective autophagy in primary hippocampal neuron with DYNC111 knockdown (Fig. 4), which is favorable for structural and functional integrity of neuron.

Both RAS-RAF and PI3K-AKT pathways are necessary for dendritic morphogenesis (Kumar et al., 2005) and neuron survival (Mazzoni et al. 1999). Additionally, PI3K-AKT signaling has a major role in antiapoptotic function (Brunet et al., 2001). Here, we demonstrate that only RAS-RAF-MEK pathway has the protective role in dendritic atrophy cause by DYNC111 knockdown (Fig. 6). Interestingly, it is recently reported that inhibition of RAS-MAPK pathway has a role in longevity of Drosophila (Slack et al., 2015). However, to avoid compromising brain function of higher animals (Fig. 24), our results raise caution about inhibiting RAS-RAF-MEK signaling for longevity. Thus, it deserves further studies on the roles of RAS-RAF-MEK signaling in longevity and neuronal atrophy with models of higher animals, such as rodents or monkeys.

\section{A more general role of RAS-RAF-MEK signaling in neuronal atrophy}

In the past decades, dominated researches and drug developments have focused or based on cholinergic hypothesis or the amyloid cascade hypothesis for 
Alzheimer's disease (Becker et al., 2008; Craig et al., 2011; Karran et al., 2011). However, the continual failures of clinical trials for neurodegenerative disorders suggest that it is important and necessary to think about new models and strategies, such as motor protein dynein malfunction and mitochondrial dysfunction, both of which are in the spotlight of neurodegeneration therapy, ALS in particular (Eschbach and Dupuis, 2011; Banks and Fisher, 2008; Moreira et al., 2010; Payne and Chinnery, 2015).

Here, based on the cellular models of neuronal atrophy, we demonstrate that BRAF has a general protective role in neuronal atrophy caused by dynein malfunction or mitochondrial impairment (Fig. 6). Dynein malfunction by DYNC1I1 knockdown decreases mitochondrial motility (Fig. 4), which may augment mitochondrial pathology. This study provides some missing linkages among dynein, mitochondria, and atrophy/degeneration. These findings about the RAS-RAF-MEK pathway for neuronal atrophy protection provide a therapeutic intervention signaling against the onsets of neuronal atrophy caused by dynein malfunction or mitochondrial impairment. Importantly, neuronal atrophy is not only the hallmark of neurodegeneration, such as ALS, but also a continuous process in adult brain with increasing age (Pakkenberg et al., 2003; Fox and Schott, 2004). Therefore, more speculatively, it might even imply a potential target to ameliorate memory decline due to age-related brain atrophy.

\section{MATERIALS AND METHODS}

Additional information can be found in the supplemental materials and methods.

\section{Plasmids and shRNA}

The isoforms of Dync1i1 are cloned into the vector pEGFP-N1 with restriction sites EcoRI and BamHI (NEB). The shRNA1-6 oligonucleotide for Dync1i1 (5'-GCATGGAGCTGGTGTACAA-3') and control shRNA1-4 oligonucleotide (5'-GCTGGAGCCAACCTTTCTT-3') are constructed into the modified pSUPER vector $\left(\mathrm{Kan}^{r}\right.$, EGFP expression) with Bg/ll and Hindlll restriction sites. The calcium phosphate method is used for transfection.

\section{Data and statistical analysis}

Imaging is performed using an Olympus FV1000 confocal microscope with a $40 \times / 0.95$ objective (Olympus) for dendritic length imaging and a $60 \times / 1.2 \mathrm{w}$ objective (Olympus) for high-resolution imaging at room temperature or mitochondrial motility study at $37^{\circ} \mathrm{C}$. The sholl analysis of neuritic morphology and complexity is performed using Fiji/lmageJ software with Simple Neurite Tracer. Photos of GFP-LC3 labeled autophagosomes are imaged with confocal microscope and further deconvolved using the SharpStack Total Deconvolution function of Image-Pro Plus (Media Cybernetics). The numbers of autophagosomes are counted by triple-blinded analysis. Data sets in sholl-analysis graphs are presented as mean \pm SEM from repeats in at least three independent experiments, while data sets in text are presented as mean \pm SD. Scatterplots with boxplots are plotted with $\mathrm{R}$ software. For mitochondrial motility study, motile and stationary dendritic mitochondria are separated with an immobile filter, which is computed with FFT algorithm. When comparing multiple samples in a group, one-way ANOVA test is used. When comparing two samples, two-tailed Student's $t$ test is used.

\section{ACKNOWLEDGEMENTS}

We thank the following people for their help: Dr. Huai-Bin Cai for critical reading of the manuscript; Dr. Kevin Pfister, Dr. Yan Chen, Dr. Zheng Li, and Dr. Zun-Ji Ke for sharing experimental materials. This work was supported by the National Natural Science Foundation of China (Grant No. 31171369), and partially supported by the National Basic Research Program (973 Program) (Nos. 2011CB910903 and 2010CB912001), Chinese Academy of Sciences (Hundred Talents Program and 2009OHTP10).

\section{ABBREVIATIONS}

$A D$, Alzheimer's disease; ALS, amyotrophic lateral sclerosis; HD, Huntington's disease; MAPK, mitogen activated protein kinase; PD, Parkinson's disease; PI3K, phosphoinositide 3-kinase; PKB/AKT, Protein kinase B; TMRE, tetramethylrhodamine ethyl ester; TMRM, tetramethylrhodamine methyl ester.

\section{COMPLIANCE WITH ETHICAL STANDARDS}

Zhi-Dong Liu, Su Zhang, Jian-Jin Hao, Tao-Rong Xie, and JianSheng Kang declare that they have no conflict of interest.

All institutional and national guidelines for the care and use of laboratory animals were followed.

\section{AUTHOR CONTRIBUTIONS}

Z.-D. L and S. Z designed and conducted the experiments and manuscript writing. J.-J. H and T.-R. X did biochemistry experiments and data analysis. J.-S. K developed the idea, directed the study and wrote the paper. All authors participated in discussions.

\section{OPEN ACCESS}

This article is distributed under the terms of the Creative Commons Attribution 4.0 International License (http://creativecommons.org/ licenses/by/4.0/), which permits unrestricted use, distribution, and reproduction in any medium, provided you give appropriate credit to the original author(s) and the source, provide a link to the Creative Commons license, and indicate if changes were made.

\section{REFERENCES}

Banks GT, Fisher EM (2008) Cytoplasmic dynein could be key to understanding neurodegeneration. Genome Biol 9:214

Becker RE, Greig NH, Giacobini E (2008) Why do so many drugs for Alzheimer's disease fail in development? Time for new methods and new practices? J Alzheimers Dis 15:303-325 
Boylan KLM, Hays TS (2002) The gene for the intermediate chain subunit of cytoplasmic dynein is essential in Drosophila. Genetics 162:1211-1220

Brunet A, Datta SR, Greenberg ME (2001) Transcription-dependent and -independent control of neuronal survival by the PI3K-Akt signaling pathway. Curr Opin Neurobiol 11:297-305

Chao MV (2003) Neurotrophins and their receptors: a convergence point for many signalling pathways. Nat Rev Neurosci 4:299-309

Chen X-J, Levedakou EN, Millen KJ, Wollmann RL, Soliven B, Popko B (2007) Proprioceptive sensory neuropathy in mice with a mutation in the cytoplasmic dynein heavy chain 1 gene. J Neurosci 27:14515-14524

Chin PC, Liu L, Morrison BE, Siddiq A, Ratan RR, Bottiglieri T, D'Mello SR (2004) The c-Raf inhibitor GW5074 provides neuroprotection in vitro and in an animal model of neurodegeneration through a MEK-ERK and Akt-independent mechanism. J Neurochem 90:595-608

Craig LA, Hong NS, McDonald RJ (2011) Revisiting the cholinergic hypothesis in the development of Alzheimer's disease. Neurosci Biobehav Rev 35:1397-1409

Dotti CG, Sullivan CA, Banker GA (1988) The establishment of polarity by hippocampal neurons in culture. J Neurosci 8:1454-1468

Eschbach J, Dupuis L (2011) Cytoplasmic dynein in neurodegeneration. Pharmacol Ther 130:348-363

Fiordalisi JJ, Johnson RL II, Ülkü AS, Der CJ, Cox AD (2001) Mammalian expression vectors for Ras family proteins: generation and use of expression constructs to analyze Ras family function. In: Der CJ, Balch WE (eds) Methods in enzymology. Academic Press, San Diego, pp 3-36

Fox NC, Schott JM (2004) Imaging cerebral atrophy: normal ageing to Alzheimer's disease. Lancet 363:392-394

Freeman SH, Kandel R, Cruz L, Rozkalne A, Newell K, Frosch MP, Hedley-Whyte ET, Locascio JJ, Lipsitz L, Hyman BT (2008) Preservation of neuronal number despite age-related cortical brain atrophy in elderly subjects without Alzheimer disease. J Neuropathol Exp Neurol 67:1205-1212

Galabova-Kovacs G, Kolbus A, Matzen D, Meissl K, Piazzolla D, Rubiolo C, Steinitz K, Baccarini M (2006) ERK and beyond: insights from B-Raf and Raf-1 conditional knockouts. Cell Cycle Georget. Tex 5:1514-1518

Ha J, Lo KW-H, Myers KR, Carr TM, Humsi MK, Rasoul BA, Segal RA, Pfister KK (2008) A neuron-specific cytoplasmic dynein isoform preferentially transports TrkB signaling endosomes. J Cell Biol 181:1027-1039

Hafezparast M, Klocke R, Ruhrberg C, Marquardt A, Ahmad-Annuar A, Bowen S, Lalli G, Witherden AS, Hummerich $\mathrm{H}$, Nicholson $\mathrm{S}$ et al (2003) Mutations in dynein link motor neuron degeneration to defects in retrograde transport. Science 300:808-812

Heumann R, Goemans C, Bartsch D, Lingenhöhl K, Waldmeier PC, Hengerer B, Allegrini PR, Schellander K, Wagner EF, Arendt T et al (2000) Transgenic activation of Ras in neurons promotes hypertrophy and protects from lesion-induced degeneration. J Cell Biol 151:1537-1548

Holzbaur ELF, Vallee RB (1994) Dyneins: molecular structure and cellular function. Annu Rev Cell Biol 10:339-372

Kapitein LC, Schlager MA, Kuijpers M, Wulf PS, van Spronsen M, MacKintosh FC, Hoogenraad CC (2010) Mixed microtubules steer dynein-driven cargo transport into dendrites. Curr Biol 20:290-299

Karran E, Mercken M, Strooper BD (2011) The amyloid cascade hypothesis for Alzheimer's disease: an appraisal for the development of therapeutics. Nat. Rev. Drug Discov. 10:698-712

Kimura S, Noda T, Yoshimori T (2008) Dynein-dependent movement of autophagosomes mediates efficient encounters with lysosomes. Cell Struct Funct 33:109-122

Klionsky DJ, Abdalla FC, Abeliovich $\mathrm{H}$, Abraham RT, AcevedoArozena A, Adeli K, Agholme L, Agnello M, Agostinis P, AguirreGhiso JA et al (2012) Guidelines for the use and interpretation of assays for monitoring autophagy. Autophagy 8:445-544

Kumar V, Zhang M-X, Swank MW, Kunz J, Wu G-Y (2005) Regulation of dendritic morphogenesis by Ras-PI3K-Akt-mTOR and Ras-MAPK signaling pathways. J Neurosci 25:11288-11299

Lalli G (2014) Regulation of neuronal polarity. Exp Cell Res 328:267-275

Lipka J, Kuijpers M, Jaworski J, Hoogenraad CC (2013) Mutations in cytoplasmic dynein and its regulators cause malformations of cortical development and neurodegenerative diseases. Biochem Soc Trans 41:1605-1612

Maday S, Wallace KE, Holzbaur ELF (2012) Autophagosomes initiate distally and mature during transport toward the cell soma in primary neurons. J Cell Biol 196:407-417

Mazzoni IE, Säid FA, Aloyz R, Miller FD, Kaplan D (1999) Ras regulates sympathetic neuron survival by suppressing the p53mediated cell death pathway. J Neurosci 19:9716-9727

Mitchell DJ, Blasier KR, Jeffery ED, Ross MW, Pullikuth AK, Suo D, Park J, Smiley WR, Lo KW-H, Shabanowitz J et al (2012) Trk activation of the ERK1/2 kinase pathway stimulates intermediate chain phosphorylation and recruits cytoplasmic dynein to signaling endosomes for retrograde axonal transport. J Neurosci 32:15495-15510

Moreira PI, Zhu X, Wang X, Lee H, Nunomura A, Petersen RB, Perry G, Smith MA (2010) Mitochondria: a therapeutic target in neurodegeneration. Biochim Biophys Acta 1802:212-220

Myers KR, Lo KW-H, Lye RJ, Kogoy JM, Soura V, Hafezparast M, Pfister KK (2007) Intermediate chain subunit as a probe for cytoplasmic dynein function: biochemical analyses and live cell imaging in PC12 cells. J Neurosci Res 85:2640-2647

Pakkenberg B, Pelvig D, Marner L, Bundgaard MJ, Gundersen HJG, Nyengaard JR, Regeur L (2003) Aging and the human neocortex. Exp Gerontol 38:95-99

Payne BAl, Chinnery PF (2015) Mitochondrial dysfunction in aging: much progress but many unresolved questions. Biochim. Biophys. Acta BBA - Bioenerg. 1847:1347-1353

Pfister KK, Fisher EMC, Gibbons IR, Hays TS, Holzbaur ELF, Mclntosh JR, Porter ME, Schroer TA, Vaughan KT, Witman GB et al (2005) Cytoplasmic dynein nomenclature. J Cell Biol 171:411-413

Ravikumar B, Acevedo-Arozena A, Imarisio S, Berger Z, Vacher C, O'Kane CJ, Brown SDM, Rubinsztein DC (2005) Dynein mutations impair autophagic clearance of aggregate-prone proteins. Nat Genet 37:771-776

Regeur L, Badsberg Jensen G, Pakkenberg H, Evans SM, Pakkenberg B (1994) No global neocortical nerve cell loss in brains from patients with senile dementia of Alzheimer's type. Neurobiol Aging 15:347-352 
Rosse C, Boeckeler K, Linch M, Radtke S, Frith D, Barnouin K, Morsi AS, Hafezparast M, Howell M, Parker PJ (2012) Binding of dynein intermediate chain 2 to paxillin controls focal adhesion dynamics and migration. J Cell Sci 125:3733-3738

Scaduto RC Jr, Grotyohann LW (1999) Measurement of mitochondrial membrane potential using fluorescent rhodamine derivatives. Biophys J 76:469-477

Schott JM, Fox NC, Frost C, Scahill RI, Janssen JC, Chan D, Jenkins R, Rossor MN (2003) Assessing the onset of structural change in familial Alzheimer's disease. Ann Neurol 53:181-188

Slack C, Alic N, Foley A, Cabecinha M, Hoddinott MP, Partridge L (2015) The Ras-Erk-ETS-signaling pathway is a drug target for longevity. Cell 162:72-83

Song A, Wang D, Chen G, Li Y, Luo J, Duan S, Poo M (2009) A selective filter for cytoplasmic transport at the axon initial segment. Cell 136:1148-1160

Soo KY, Farg M, Atkin JD (2011) Molecular motor proteins and amyotrophic lateral sclerosis. Int J Mol Sci 12:9057-9082

Swaab DF, Hofman MA, Lucassen PJ, Salehi A, Uylings HBM (1994) Neuronal atrophy, not cell death, is the main hallmark of Alzheimer's disease. Neurobiol Aging 15:369-371
Vaughan PS, Leszyk JD, Vaughan KT (2001) Cytoplasmic dynein intermediate chain phosphorylation regulates binding to dynactin. J Biol Chem 276:26171-26179

Wang J, Whiteman MW, Lian H, Wang G, Singh A, Huang D, Denmark T (2009) A non-canonical MEK/ERK signaling pathway regulates autophagy via regulating beclin 1 . J Biol Chem 284:21412-21424

Xie Y, Zhou B, Lin M-Y, Wang S, Foust KD, Sheng Z-H (2015) Endolysosomal deficits augment mitochondria pathology in spinal motor neurons of asymptomatic fALS mice. Neuron 87:355-370

Yamaguchi T, Kakefuda R, Tajima N, Sowa Y, Sakai T (2011) Antitumor activities of JTP-74057 (GSK1120212), a novel MEK1/ 2 inhibitor, on colorectal cancer cell lines in vitro and in vivo. Int $\mathrm{J}$ Oncol 39:23-31

Zheng Y, Wildonger J, Ye B, Zhang Y, Kita A, Younger SH, Zimmerman S, Jan LY, Jan YN (2008) Dynein is required for polarized dendritic transport and uniform microtubule orientation in axons. Nat Cell Biol 10:1172-1180

Zhong J, Li X, McNamee C, Chen AP, Baccarini M, Snider WD (2007) Raf kinase signaling functions in sensory neuron differentiation and axon growth in vivo. Nat Neurosci 10:598-607 